le portiQue $\begin{array}{ll}\text { Le Portique } \\ \text { Revue de philosophie et de sciences humaines }\end{array}$

33 | 2014

Straub !

\title{
Débat « Modernes/Classiques »
}

Jean-Marie Straub, David Faroult, Olivier Goetz et Jean-Marc Leveratto

\section{OpenEdition}

\section{Journals}

Édition électronique

URL : http://journals.openedition.org/leportique/2776

DOI : $10.4000 /$ leportique.2776

ISSN : $1777-5280$

\section{Éditeur}

Association "Les Amis du Portique"

Édition imprimée

Date de publication : 1 mai 2014

ISSN : 1283-8594

\section{Référence électronique}

Jean-Marie Straub, David Faroult, Olivier Goetz et Jean-Marc Leveratto, « Débat « Modernes/ Classiques » », Le Portique [En ligne], 33 | 2014, document 3, mis en ligne le 05 février 2016, consulté le 12 avril 2021. URL : http://journals.openedition.org/leportique/2776 ; DOI : https://doi.org/10.4000/ leportique. 2776

Ce document a été généré automatiquement le 12 avril 2021.

Tous droits réservés 


\title{
Débat « Modernes/Classiques »
}

\author{
Jean-Marie Straub, David Faroult, Olivier Goetz et Jean-Marc Leveratto
}

1 Jean-Marc Leveratto - Avant d'entrer dans le vif du débat, je voudrais dire quelques mots sur le thème qui nous a été proposé par les organisateurs. Ce thème - Classiques/ Modernes - est sans doute un peu périlleux. Mais, il est une bonne manière d'introduire à un débat qui portera, au-delà des films qui ont été projetés, sur l'ensemble de l'œuvre de Jean-Marie Straub et de Danièle Huillet, et prendra en compte des films qui sont devenus des références cinématographiques.

Trois pistes de discussion s'offrent à nous. La plus évidente, c'est effectivement celle de l'adaptation de "Classiques », soit, comme Jean-Marie Straub l'a rappelé, le fait que certains des films qu'on vient de voir nous offrent l'occasion de découvrir ou de redécouvrir des œuvres d'auteurs considérés comme des génies de la littérature, de l'art et de la musique. C'est un choix artistique fort que cette volonté de porter au cinéma certaines œuvres de grands noms de la littérature universelle, de la peinture ou de la musique savante. La vision de ces films suscite immédiatement des réactions quant au traitement, souvent étonnant, voire spectaculaire, des œuvres sélectionnées. La question, traditionnelle, du «respect de l'œuvre» et de sa pertinence pour comprendre une œuvre singulière comme celle des Straub se pose alors.

Une deuxième piste de discussion est celle des "Classiques modernes ", un oxymoron couramment utilisé aujourd'hui pour désigner les classiques $\mathrm{du} \mathrm{xx}^{\mathrm{e}}$ siècle, les œuvres considérées aujourd'hui comme des références incontournables pour quiconque s'intéresse à l'art moderne. Sous cet angle, l'œuvre des Straub fait partie des "Classiques modernes", et nous donne à lire leur propre rapport à ces classiques modernes que sont les grands réalisateurs de cinéma. Un intérêt particulier, pour moi, de ce rapport des Straub au patrimoine cinématographique $d u x^{e}$ siècle réside dans l'importance qu'ils accordent au cinéma classique hollywoodien. De quelle manière les classiques du cinéma, et lesquels, sont présents dans les films qu'on vient de voir, de quelle manière les films de Straub communiquent avec d'autres grandes œuvres de l'histoire du cinéma constituerait donc une deuxième piste de discussion.

La troisième est celle, un peu énigmatique, du sentiment que nous avons tous éprouvé, je pense, le sentiment d'une résistance de l'œuvre des Straub à la modernité, au sens de 
Baudelaire, d'une capacité de leur œuvre à échapper à la mode. La formulation "Classiques/Modernes » exprime alors le sentiment de balancement du spectateur, sa difficulté à choisir le terme adapté pour parler de ce qu'il vient de voir. Classique ou moderne ? La formule devient une invitation à penser, non seulement la polysémie du terme de modernité, mais une forme d'antimodernisme qui est présent dans ce qu'on a $\mathrm{pu}$ voir lors de cette rétrospective. Une mise à distance de la modernité, pour réaffirmer un lien avec une tradition mais aussi pour s'ouvrir à ce qui est en train d'advenir. J'espère que cette troisième piste de discussion - de quelle manière décrire justement ce qu'on a vu ? de quelle manière exprimer la singularité esthétique - offrira l'occasion à toutes les personnes présentes de participer.

Olivier Goetz - Il me semble qu'il y a effectivement un piège dans l'intitulé de ce débat, Classiques/Modernes. Ce sont des mots qui veulent tout dire et, en même temps, ne veulent rien dire du tout. Tout dépend du sens qu'on leur donne. C'est le piège inhérent à l'approche générale choisie par Ciné Art, au choix d'une organisation thématique de cette rétrospective...

6 Jean-Marie Straub - Mais non, c'était le souhait du Centre Pompidou-Metz !

7 O. G. - Dont acte. Jean-Marie Straub était, en effet, opposé à cette approche thématique, comme il vient de le rappeler. Une approche chronologique aurait été mieux adaptée à une « rétrospective ». Mais il faut quand même remercier le Centre Pompidou-Metz de nous avoir accueilli et, surtout, Ciné Art pour avoir organisé cette rétrospective. Elle nous a permis non seulement de voir des films, mais de voir une œuvre. Car c'est bien le sens et l'intérêt d'une rétrospective que de nous faire découvrir une œuvre dans sa globalité.

J.-M. S. - Les institutions culturelles ne s'intéressent pas aux œuvres. Ça n'existe pas pour eux. Ce qui les intéresse, c'est les thèmes. Comme Arte.

9 O. G. - Jean-Marie, il me semble justement que le fait que tu sois là, présent parmi nous, change radicalement le regard qu'on porte sur les films. On voit les films autrement. En même temps, ce regard privilégié possède tout de même une limite... Puisque nous sommes au Centre Pompidou, je peux citer une divinité pompidolienne, Marcel Duchamp, qui nous rappelle que c'est le regardeur qui fait le tableau. C'est dire que les spectateurs font aussi les films, à leur façon. Qu'il y a un moment où le réalisateur n'est plus totalement le maître du sens de ses films. Et qu'on peut donc accepter cette approche thématique qui nous est proposée, même si on la trouve un peu biaisée.

10 Je l'accepte d'autant plus volontiers que cette problématique Classiques versus Modernes est présente - Jean-Marc Leveratto vient de le rappeler - dans le discours de Jean-Marie Straub et Danièle Huillet. Ils ont souvent parlé de la question du moderne ou de la modernité. On ne sait jamais très bien, cependant, quelle modernité ils visent.

11 Par exemple, on découvre, en regardant Du jour au lendemain, que la "mode » est une cible de cet opéra d'Arnold Schönberg. Cela nous rappelle que le mot moderne, qui peut être un très beau mot, peut être également un mot très vilain, lorsque la télévision, par exemple, nous inonde de publicité pour des lessives modernes ou d'autres choses, présentées comme désirables parce que «modernes »... Tout le monde sait que le mot moderne vient du latin, modo, qui veut dire « récemment». Le moderne, en un sens, c'est ce qui est récent. Mais c'est aussi, comme on sait, la caractéristique des périodes historiques qu'on appelle modernes ou même, aujourd'hui, "postmodernes». Ce qui signifierait qu'il existe une modernité plus moderne que le moderne... Et pourquoi pas? 
Évitons simplement de nous enfermer dans des questions de terminologie pour parler de l'œuvre.

David Faroult - Je voudrais commencer par remercier mes collègues qui sont à la table et les organisateurs de m'avoir invité. D'abord, parce que j'éprouve beaucoup de plaisir à être là. Ensuite, parce que je crains d'être un peu déloyal à leur égard en prenant mes distances par rapport au thème proposé pour le débat. Je vais essayer de dire pourquoi !

La première raison, c'est la polysémie de ces termes de modernité et de classicisme. Par exemple, il n'est pas possible, d'un point de vue stylistique, de définir Hölderlin comme un poète classique. On le caractérise généralement comme un romantique. Cette taxinomie Classiques/Modernes ne nous aide donc pas vraiment à penser ce qui se passe entre nous et l'œuvre de Jean-Marie Straub et Danièle Huillet. De la même façon, la triade classique, moderne, postmoderne, désigne des périodes historiques, des époques artistiques. L'œuvre de Jean-Marie Straub et Danièle Huillet, en ce sens, s'étend entre la fin d'une période moderne et le début d'une période postmoderne.

14 Je crois donc que ces mots n'aident pas du tout à caractériser ce qui se passe dans l'œuvre, ce qui se passe dans les films. Parce que justement, et heureusement, ces films sont largement à contretemps à l'égard de leur époque. Je pense que c'est surtout ça qui fait qu'on peut entretenir des liens particulièrement étroits ou intenses avec eux.

Pour la suite, pour tout que je pourrais être amené à dire dans la discussion, je propose de distinguer, un peu grossièrement, deux types de films. Les uns, qui sont la majorité écrasante, qui s'adressent toujours à la même masse d'un public indifférencié et dont le projet est de faire vibrer cette masse d'un public indifférencié à l'unisson, avec les mêmes émotions, au même moment. Les autres, très minoritaires, mais qui sont peutêtre ceux sur lesquels on a le plus envie de travailler quand on s'intéresse au cinéma ou quand on travaille sur le cinéma, qui s'adressent à chaque spectateur. Des films qui s'adressent à chaque spectateur, singulièrement, et peut-être des films qui savent - en tout cas dont les auteurs savent - l'extrême et irréductible solitude dans laquelle on peut se trouver face à un film, même dans une salle pleine. Il me semble que le cinéma de Jean-Marie Straub - qui n'est pas le seul à cet égard - appartient à cette deuxième catégorie.

16 Je ressens donc un certain embarras à prétendre prononcer quoi que ce soit qui puisse prétendre être objectif à l'égard du cinéma de Jean-Marie Straub. C'est que tout au plus, je me sentirai capable de dire ce que cette œuvre représente pour moi ou pourquoi elle compte pour moi. Comment exprimer en quelques mots pourquoi je me sens si proche de l'œuvre de Jean-Marie Straub ? C'est un cinéma, et peut-être une personne aussi, qui combinent en même temps une immense amicalité égalitaire et un caractère terriblement intimidant.

"L'amicalité ", au sens où Brecht emploie très souvent ce terme, c'est adresser à l'autre en même temps une immense bienveillance et une redoutable exigence. Je trouve que la personne et les films de Jean-Marie Straub nous adressent cette bienveillance et cette exigence à chaque instant. Et que cette exigence, justement, peut avoir un caractère terriblement intimidant. C'est là qu'il y a peut-être un paradoxe, un oxymore auquel, effectivement, il faut tenir pour essayer de rendre justice à ce qui se passe devant les films de Jean-Marie Straub. C'est le sentiment qu'en même temps, ces films nous invitent à un rapport rigoureusement égalitaire, et qu'en même temps, ils le font dans des modalités qui peuvent être terriblement - au sens de la terreur - intimidantes. Pourquoi égalitaire? Parce que ce cinéma se démarque de celui qui s'adresse à nous 
comme à une masse indifférenciée, en cherchant à nous mâcher les émotions et à nous mâcher le travail à tout instant. De sorte qu'on n'a jamais de troubles ou de questions à se poser pour savoir ce qu'on doit penser de ce qui se dit, ce qu'on doit penser d'un personnage, ce qu'on doit penser de la situation à un instant donné. Alors que dans le cinéma dominant, on ne cesse pas de décider pour nous, à l'avance, quand est-ce qu'on doit être tendu ou détendu, rire ou pleurer, etc.

$\mathrm{Au}$ contraire, dans le cinéma de Jean-Marie Straub, c'est à notre expérience de la vie qu'il est fait appel, pour savoir ce qu'on en pense à chaque instant. Donc, on devient un peu - pour le dire simplement - le coréalisateur du film, par le travail de réception, ce que rappelait Olivier Goetz. Et à ce titre-là, on est invités dans l'équipe à un titre égalitaire. Le caractère intimidant de la situation ne réside peut-être pas dans les films eux-mêmes, mais dans le contexte dans lesquels on les reçoit, celui de nos habitudes de spectateur - Nous ne sommes pas du tout habitués à être renvoyés à nous-mêmes, pour savoir ce qu'on en pense à chaque instant. Et cela peut être réellement très intimidant. Je crois qu'il y a derrière ces films une conception de l'égalité amicale, avec cette dose d'exigence inhérente à une égalité qui n'est pas au rabais. De même que le communisme dont se réclame Straub est un communisme non populiste, antiproductiviste ou anti-industrialiste, résolument internationaliste et antimilitariste d'ailleurs Straub dans sa vie je crois l'a payé assez cher -, je crois que cette amicalité c'est comme ça que je l'ai vécue - aide à s'élever, pour ceux qui veulent bien en suivre l'invitation.

19 Je me souviens de Jean-Marie Straub répondant à une spectatrice, qui se plaignait de s'être ennuyée pendant un film, « on est responsable de son ennui !». Sur le moment, j'avais trouvé cette réponse très dure. Avec le temps, j'y adhère de plus en plus. Je crois qu'on est toujours libre de ne pas répondre à une invitation amicale, mais s'en plaindre est quelque chose de terriblement arrogant, je trouve...

J.-M L. - Comme David Faroult vient de le souligner, cette rétrospective a été en effet l'occasion de nous confronter à un cinéma qui est exigeant au sens où il demande aux spectateurs de participer. C'est ce que j'ai ressenti dans toutes les occasions, puisque j'ai vu des films des Straub à différentes périodes de ma vie, où j'ai accepté l'épreuve que constitue leur vision. Mais, cette fois-ci, et pour revenir au thème qui nous a été proposé, j'ai été frappé, en découvrant Othon, par la dimension humaniste de ce cinéma. Il nous invite souvent à nous intéresser à des œuvres qui sont des œuvres classiques au sens où elles sont enseignées à l'école. Nous avons tous fait, je parle pour les spectateurs de ma génération, notre pensum sur Corneille, même si nous n'avons pas lu Othon. Le film - comme le dit la dédicace de la fin - nous offre l'occasion de nous mesurer avec un texte de Corneille et permet aux spectateurs de vérifier qu'ils ne sont pas aussi bêtes qu'ils peuvent le croire, parfois, s'ils n'ont pas fait d'études. C'est un film qui m'a touché parce qu'il m'a intéressé à un texte pour lequel au départ je n'avais aucune appétence. Mon expérience vérifie donc ce que David Faroult vient de dire de l'ensemble des films des Straub. Ils ne prennent pas les spectateurs pour des idiots culturels, pour reprendre la formule d'un sociologue américain. Ils se refusent à considérer le spectateur comme une personne incapable de curiosité et d'effort d'appréhension, comme une personne qui serait par définition étrangère tant à la culture qu'à la culture cinématographique. Le film m'a fait non seulement voir mais entendre, au double sens du terme, Othon de Pierre Corneille. Du coup, je ne suis pas du tout d'accord avec la réaction d'un spectateur - je ne sais pas s'il est présent dans la 
salle - qui a dit que le film avait réussi à rendre la langue de Corneille incompréhensible. C'est vous?

21 Spectateur - Je n'ai pas dit la langue de Corneille. J'ai dit que ma langue maternelle me devenait tout d'un coup incompréhensible. Parce que le film me révélait une étrangeté dans cette langue, que je découvrais comme si je ne la comprenais pas. Ce n'était pas uniquement celle de Corneille.

J.-M. L. - Merci pour cette précision. Elle réactive le souvenir que j'ai gardé du premier film que j'ai vu de Jean-Marie Straub et Danièle Huillet. Il s'agit de Leçon d'histoire, une adaptation des Affaires de Monsieur Jules César, le roman de Berthold Brecht. Ce souvenir est très contrasté, celui d'une chose que j'ai trouvée trop lourde, trop longue et, en même temps, assez attachante puisque j'en conserve encore aujourd'hui une vive impression. D'une espèce de plaisir très simple, éprouvé à la vision de ce sénateur en train de raconter la carrière de Monsieur Jules César en buvant un verre de rouge attablé à une terrasse de la Rome d'aujourd'hui. Un plaisir exemplaire de l'ironie brechtienne, extrêmement efficace car appliquée à Brecht lui-même, à quelqu'un qui est lui-même devenu un classique du marxisme. Un plaisir dans la continuité de ce que lui-même a prôné, qu'on doit ne jamais oublier que le temps de la réception n'est pas le temps de la production, et qu'on a le droit de mettre en relation la Rome impériale et le monde moderne, la cité et la city, le Sénat et la Chambre des députés, etc., parce ce qui nous importe, en tant que spectateurs citoyens, c'est de penser la manière dont ces choses nous concernent tous aujourd'hui. C'est un bon exemple de la capacité des films de Straub à situer les choses dont ils parlent dans le temps contemporain, à les mettre en relation avec des choses que l'on vit, à nous aider à les critiquer. C'est ce que David Faroult a pointé en parlant d'un communisme des Straub.

Spectateur - Hier soir, j'ai repensé à cette histoire d'Othon. C'est un des films que j'adore. C'est la deuxième fois que je le voyais. La première fois, il m'avait beaucoup frappé. Et cette fois-ci encore. Dans la nuit, je me suis réveillé avec Othon en tête. C'était en rapport avec ce que JeanMarie Straub nous a dit hier sur la modernité de Corneille. Ce qui faisait que Corneille était si moderne, c'était qu'à la différence de la tragédie, ses personnages étaient aussi perdus sur la question de leur destinée que le public. Je me suis dit que le film de Straub accentuait cette modernité. Ce que je préfére, dans Othon, c'est que le drame se joue au niveau de la langue, et au niveau de la langue traitée en tant que matière, et pas seulement en tant que moyen d'exposer une intrigue policière, comme il l'a dit. En ce qui concerne l'intrigue policière-parce que je suis un peu inculte et que je n'ai jamais lu Corneille - j'étais perdu. Mais là, le drame se jouait vraiment dans ces rapports de diction, de langue et de matière même de la langue. C'est là que j'ai vu le drame et ressenti la possibilité de réactiver la modernité.

24 O. G. - Jean-Marc Leveratto vient de définir le classique comme ce qu'on apprend à l'école, en classe. Et c'est vrai que presque tous les films des Straub sont faits à partir d'un objet textuel qu'on peut étudier à l'école, qu'on peut étudier à l'université. Pour autant, ce sont aussi, et toujours, des œuvres qui sont un peu décalées par rapport à ce qu'on apprend vraiment à l'école. Par exemple, Jean-Marc Leveratto l'a signalé, le Corneille qu'ils ont choisi de filmer, ce n'est pas Le Cid, c'est Othon, une pièce méconnue. Jean-Marie Straub a tenu à nous citer, hier, le Petit Larousse qui disait que c'était une pièce qu'il ne fallait pas lire, car c'était une mauvaise pièce de Corneille. Si l'on considère le Petit Larousse comme un instrument de mesure du classicisme, on voit qu'Othon ne peut pas être considéré comme un classique. On peut dire la même chose de tous les autres auteurs qui sont mis en scène par les Straub, Kafka, Mallarmé, etc. Mallarmé, par exemple, ce n'est certainement pas un classique, c'est un moderne dans 
le sens où Rimbaud dit qu'il faut être moderne... Dans ce sens, je ne crois pas qu'il n'y ait un seul texte qui soit vraiment classique à proprement parler. Mais si classique veut dire respect plutôt que découpage, charcutage, tripatouillage, il y a chez les Straub, en effet, un respect infini des textes, qui s'accompagne d'un respect infini des images. Cela a souvent été dit pendant la rétrospective : respect du son direct, respect du cadrage, une éthique de la technique, de l'artisanat du cinéma. Le résultat un peu paradoxal de cet incroyable respect de tout est que cela produit une étrangeté qui entraîne parfois des réactions comme celle que vient d'exprimer ce spectateur, l'impression que c'est tellement étranger que ce n'est plus ce qu'on croit que c'est. On est dépaysé, on est déplacé. Mon impression par rapport à Othon est plutôt inverse de celle exprimée par le spectateur qui s'est exprimé. On comprend absolument tout, même si on éprouve le sentiment de n'y comprendre rien. C'est au point que moi, qui ne parle pas allemand, j'ai l'impression de savoir l'allemand en regardant Schwartz Sünde, parce que c'est tellement dit que ça en devient limpide. C'est la conjugaison paradoxale d'un rapprochement et d'un éloignement. C'est ce qui fait que ce film donne l'impression d'être plus classique que les classiques, en tout cas que les classiques enseignés par nos professeurs d'école. N'oublions pas, par ailleurs, ce qui a déjà été pointé. Parler ici de classiques, ce n'est pas seulement se référer à des textes littéraires, c'est aussi mobiliser de grandes œuvres de l'histoire de l'art cinématographique.

D. F. - C'est en ce sens que Godard disait : « Nous sommes les derniers des classiques ». Ce qui contribue à brouiller les cartes, c'est donc aussi le fait que Jean-Marie Straub ait choisi, pour la programmation cinématographique qui accompagne cette rétrospective, des films qui sont tenus pour des classiques du cinéma. C'est un autre aspect du thème Classiques/Modernes dans lequel, il me semble, nous devons éviter de nous enfermer si nous voulons comprendre l'œuvre des Straub.

Francis Guermann (Ciné Art) - En tant qu'organisateur, j'assume tout à fait la construction thématique. C'est bien notre choix, ce n'est pas le Centre Pompidou-Metz qui nous l'a imposé. C'est vrai qu'au départ, notre idée était de passer tous les films par ordre chronologique sur une période de quatre mois. Simplement nous n'avons pas pu la mettre en œuvre. Le thème qui vous a été proposé, ceci dit, n'est qu'un prétexte à l'échange et vous avez toute latitude de dire ce qui vous convient.

O. G. - Je l'ai dit déjà tout à l'heure, il y a quelque chose qui me semble, dans le discours des Straub, coller à cette thématique, qui est parfois la détestation de la mode, d'une modernité qui est agressive et qui met par exemple le héros d'Amerika dans des situations absolument terrifiantes et qui est aussi cette modernité qui amène les catastrophes du Japon aujourd'hui ou le fait que, quand il va filmer la montagne SainteVictoire, elle a brûlé la veille. Il y a quelque chose d'une revendication, d'une haine de ce moderne-là, chez les Straub. Mais ce serait à Jean-Marie de la dire plutôt qu'à moi à sa place. Il m'est venu à l'esprit le petit texte de Michel Foucault sur « Qu'est-ce qu'un auteur?». Foucault y oppose la modernité, en tant que rapport à l'origine, à la tradition qui génère de la similitude, de la copie, qui se situe dans une sorte de transmission presque automatique des choses, et qui se rapproche un peu d'une certaine production éditoriale ou cinématographique dont parlait David Faroult. Dans la modernité d'aujourd'hui, dans ce qu'il peut y avoir de plus avancé dans la poésie, la musique et la littérature ou la philosophie, depuis la guerre, Foucault remarque qu'à la pointe extrême de la création, il y a toujours cette tendance d'un retour à l'origine. Il parlait de Freud, de Marx et d'autres auteurs. Il me semble qu'un certain nombre de 
textes ou d'œuvres qui ont inspiré Jean-Marie Straub et Danièle Huillet relèvent un peu de ce retour à l'origine qui a inspiré un certain nombre de créateurs de l'après-guerre, ou du moins qui les a vraiment interrogés. Il y a bien sûr Hölderlin, qui a été redécouvert au début $\mathrm{du} \mathrm{xx}^{\mathrm{e}}$ siècle par toute une série de poètes, mais qui a été lu ensuite par Heidegger et un certain nombre de philosophes. Il y a bien sûr Mallarmé qui a beaucoup inspiré un certain nombre de poètes. Il y a aussi Schönberg pour la musique, qui est un peu un point de départ pour toute une série de compositeurs de l'après-guerre. Il y a un ensemble d'œuvres ou de textes qui semblent effectivement relever de cette tentation d'un retour à l'origine que Foucault avait relevé dans certaines formes de modernité. Il y a deux éléments qui me semblent intéressants dans cette idée du retour à l'origine. Au lieu de générer de la similitude, il génère de l'écart et de la différence. Et au lieu de nous situer dans une transmission un peu bête et facile des choses, il renvoie à une sorte d'oubli fondamental. À l'époque où Foucault a écrit son texte, il parlait surtout de Freud, dont on relisait sans arrêt les œuvres parce qu'on s'apercevait qu'on ne savait pas les lire et qu'il y avait un sens littéral qui échappait assez systématiquement aux lectures de l'époque. Cette observation me semble renvoyer à un certain nombre d'œuvres qui sont en résonance avec le cinéma de Straub et Huillet. Peut-être un peu moins avec Corneille. Justement, la modernité d'aprèsguerre, c'était plutôt Racine que Corneille. Mais il y une résonance, en tout cas, avec toutes les œuvres qu'on a vues depuis une semaine.

J.-M. L. - Quelle que soit la polysémie des termes de classiques et de modernes, il semble difficile d'y échapper, surtout lorsqu'on parle d'histoire du cinéma. Une présentation des « classiques du cinéma » s'impose dans tout enseignement du cinéma, à savoir des films que les étudiants doivent connaître pour pouvoir mieux comprendre des films contemporains, parce qu'ils partagent des exigences artistiques communes. Par là, les classiques du cinéma peuvent nous aider à rendre compte de l'émotion qu'on ressent en regardant certains des films des Straub aujourd'hui. Par exemple, en revoyant Lothringen!, lors de cette rétrospective, je me suis amusé à y reconnaître un film fordien. Jean-Marie Straub a certainement dû le redire ou le suggérer, ou y faire référence dans la discussion, et j'ai vérifié qu'il ne s'agit pas d'une plaisanterie. J'ai retrouvé effectivement une émotion similaire à celle ressentie devant Qu'elle était verte ma vallée, quand j'ai revu Lothringen!, il y a quelques jours. Cela éclaire les références à Ford qui reviennent souvent dans le discours de Jean-Marie Straub, que je prenais pour des coquetteries, et montre qu'il faut les prendre au sérieux. Par seulement parce qu'il l'a dit, mais parce que l'œuvre des Straub s'inscrit vraiment dans une continuité avec le grand cinéma classique, non seulement européen, mais aussi hollywoodien, des années 30-50.

29 J.-M. S. - Il a une satire de Schönberg qui est une satire chantée, ça s'appelle Klassisch, nicht Klassisch, Klassisch, nicht Klassish. Écoutez ça et vous verrez, vous serez ravi! Par ailleurs, j'ai seulement à dire deux choses obsédantes que j'ai citées beaucoup trop souvent. Il y a premièrement que la modernité, c'est le saut du tigre dans le passé. Deuxièmement, faire la révolution, c'est aussi remettre en place des choses très anciennes et oubliées. Voilà, c'est tout !

O. G. - Ce sont des citations empruntées à Walter Benjamin, mais on peut trouver aussi chez Hannah Arendt des phrases équivalentes. Jean-Marie, peut-être pourrais-tu répondre à une question? Quand tu parles de "faire le saut du tigre dans le passé », on a toujours l'impression que tu convoques la paysannerie ou la terre. Qu'est-ce que tu 
veux dire exactement quand tu parles des paysans et quand tu dis, par exemple, que " pour faire Bach, il faut je ne sais combien de générations de paysans avant lui »? C'est un discours qui peut paraître extrêmement moderne ou extrêmement passéiste, c'est selon. Modernes. Peu importe les termes qu'on se fixe, tant qu'on discute de l'œuvre! Je crois qu'il y a peu d'œuvres cinématographiques qui se sont autant intéressées à la période de l'Antiquité. Il y a Othon (Les yeux ne veulent pas en tout temps se fermer ou Peut-être qu'un jour Rome se permettra de choisir à son tour) et Leçons d'histoire (Geschichtsunterricht), sur l'antiquité romaine; Le Corneille-Brecht récent avec l'extrait du procès de Lucullus ; Moïse et Aaron, c'est l'Antiquité biblique, disons ; La Mort d'Empédocle et Noir Péché, c'est l'Antiquité grecque; les films autour des textes de Pavese convoquent la mythologie. Cet intérêt pour l'Antiquité, qui n'a évidemment rien à voir avec le péplum ou je ne sais quel genre, est récurrent. Or, justement, depuis le temps qu'on vous entend, JeanMarie, citer cette phrase de Walter Benjamin, « La révolution, c'est le saut du tigre dans le passé », et celle de Charles Péguy, "Renouer avec des choses très anciennes mais oubliées ", on aurait pu s'attendre à vous voir renouer, comme nous y invitait Rosa Luxembourg, avec les expériences de communisme primitif, plutôt qu'avec l'Antiquité. Je trouve frappant que ce soit surtout l'Antiquité qui soit présente dans votre cinéma. Ce serait ma façon de reposer la question d'olivier Goetz.

J.-M. S. - La mythologie, ce n'est pas l'Antiquité. La mythologie, c'est l'histoire des paysans. C'est l'invention des dieux. Qui a inventé les dieux? Les paysans. La mythologie, ce n'est pas du tout l'Antiquité. Il n'y a aucune Antiquité dans la mythologie.

D. F. - Elle nous parvient de cette époque.

J.-M. S. - Ce n'est pas de ma faute ! C'est de la vôtre.

O. G. - J'avais la phrase de Walter Benjamin sous les yeux, je me permets de vous la lire : «La mode, c'est flairer l'actuel si profondément qu'il se niche dans les fourrés de l'autrefois, elle - il parle de la mode ! - est le saut du tigre dans le passé mais ceci a lieu dans une arène où commande la classe dominante. Le même saut effectué sous le ciel libre de l'histoire est le saut dialectique, la révolution telle que la concevait Marx ». Il y a donc plusieurs façons de faire le saut dans le passé. Des bonnes et des mauvaises façons. C'est comme ça que je l'interprète.

Spectateur - Où commence l'Antiquité? La mythologie s'arrête où ? Quelle est la frontière entre mythologie et Antiquité, si l'on ne doit pas confondre les deux ?...

J.-M. S. - Je ne sais pas. Eux, ils le savent. Moi, je ne sais pas.

Spectateur - Parce qu'on ne peut pas dire aussi brutalement que l'Antiquité, ce n'est pas la mythologie. Il faudrait au moins préciser la distinction?

40 J.-M. S. - Au moment où l'on a exproprié les paysans de leur histoire et de leur passé, on a dû inventer l'Antiquité. C'est la bourgeoisie. Qui a inventé l'Antiquité ? Ce sont les gens de 1789, qui ont refoulé les paysans pour s'établir eux-mêmes. Ce n'est pas compliqué. L'Antiquité commence en 1789. C'est un peu rigolo, mais c'est quand même ça. Relisez les cinq ou sept pages de Michelet sur ce qui se passait dans la vallée de la 
Saône. Je vous assure que c'est très beau et très instructif, ce qui se passait dans la vallée de la Saône un peu avant 1789 !

O. G. - Qu'est-ce qui s'est passé dans la vallée de la Saône ?...

42 J.-M. S. - Lisez Michelet! Il s'est passé que les paysans attendaient quelque chose et qu'ils se sont faits rouler. C'est tout. Il s'est passé que c'était le début de l'établissement de la bourgeoisie dans son Antiquité. Dans son humanisme. Dans son voltairianisme. Dans les banques. Dans le progrès, dans la croissance. Tout ça.

43 J.-M. L. - En parlant de films qui font référence à l'Antiquité, David, tu les opposais à quoi?

D. F. - Je ne les opposais à rien. Je disais juste qu'il y avait peu d'œuvres cinématographiques où l'Antiquité était aussi présente. Ça a l'air d'être une période, j'ai peut-être eu tort d'invoquer les Pavese là-dedans, mais ça a l'air d'être une période qui en tout cas questionne Jean-Marie Straub.

45 J.-M. L. - C'est vrai qu'on peut faire une différence entre la confrontation avec l'Antiquité dont certains films offrent l'occasion et les films qui resituent cette paysannerie dont Jean-Marie Straub vient de parler, les films qui nous situent vraiment $\mathrm{au} \mathrm{xx}^{\mathrm{e}}$ siècle, en tant que siècle des révolutions, comme disait Lénine. L'œuvre des Straub, il me semble, pose cette question de la mise du cinéma au service de cette mémoire des transformations révolutionnaires. Ce qui me touche, en tout cas, quand je regarde De la nuée à la résistance ou Sicilia, c'est la transmission d'une mémoire des luttes, à travers évidemment les personnalités littéraires de Pavese ou de Vittorini, mais des écrivains qui ont été des écrivains militants, dans le sens où leur poétique s'est voulue être à l'unisson de ceux qu'ils cherchaient à représenter. On retrouve bien la question de la mythologie telle que la pose Jean-Marie Straub chez Pavese, par la manière étonnante dont il réussit à restituer une culture paysanne, une mentalité paysanne, une vision du monde paysanne.

Il y a un auteur que je n'ai jamais entendu évoquer par Jean-Marie Straub, c'est Antonio Gramsci. Mais, parfois, ce que je ressens quand je regarde ses adaptations de Pavese ou de Vittorini, c'est une idée gramscienne, le rappel de l'existence d'une forme de sagesse, je ne sais comment dire, paysanne ou populaire, en ayant bien conscience de la manière dont ces deux termes peuvent être aujourd'hui galvaudés. Est-ce que cette question de la mémoire des luttes paysannes est moderne ? Elle est actuelle en tout cas. Elle renvoie à la question brechtienne de comment est-ce qu'on peut faire pour que les choses changent, comment montrer les choses pour qu'elles changent...

47 J.-M. S. - On oublie toujours un truc, nous tous. C'est ce qu'on a appelé les guerres de paysans, c'est-à-dire les guerres contre les paysans. On a appelé ça les guerres de paysans parce que les paysans en avaient ras-le-bol et que, tout d'un coup, ils sont sortis de leurs gonds. Les guerres de paysans, dont la plus violente a été la dernière. Les Allemands ont réussi à corrompre et à gagner et à convaincre et à entraîner les Alsaciens. Et ça a été la plus violente. Ces guerres de paysans-là, c'est le début de l'époque moderne. Parce qu'après qu'ils aient fait ce travail-là, ça a continué, jusqu'à la motoculture y compris.

48 D. F. - Je crois que parler d'une idéologie paysanne de Straub est une façon un peu trop rapide d'aborder les choses. Je parlais tout à l'heure d'une œuvre à contretemps. Et je pense qu'elle l'est pour tout le monde, y compris pour les communistes - et je parle pour moi - parce que c'est une œuvre qui nous invite à rompre avec une vision 
progressiste de l'histoire et avec cette espèce d'idéologie du progrès qui donne l'impression que l'industrialisation serait un mieux. Cette attention aux paysans, c'est aussi une attention à l'enclenchement de cette industrialisation qui a commencé à tout foutre par terre, à tout détruire. Le contretemps de l'œuvre vient aussi d'un ancrage très profond dans le temps présent. J'ai retrouvé une citation d'un entretien de JeanMarie Straub autour d'Othon qui, à mes yeux, est quasiment un programme. Je vous la relis : "Othon exige du public pour l'intéresser une intelligence sans une seconde de défaillance, tout en laissant le maximum de liberté à son imagination. La révolution a besoin d'imagination et d'intelligence et la bourgeoisie et les intellectuels sont intolérants. Tout spectateur doué de la moindre expérience morale et politique peut savoir chaque seconde du film à qui il a affaire avec chaque personnage. Tant pis pour les autres spectateurs. Les films qui prétendent leur mâcher la besogne ne leur servent quand même de rien. Ils se trouvent tout aussi désarmés dans la vie à la première occasion ».

Pour moi, c'est une déclaration programmatique en ce qu'il s'agit de faire un cinéma qui à la différence des autres films qui se font, requiert l'intelligence, requiert l'imagination, requiert un positionnement de chaque instant, précisément parce que tous les autres films, en nous rendant paresseux sur ce terrain-là, de l'intelligence, de l'imagination, du positionnement, réduisent nos facultés à cet égard. C'est en ce sens qu'il prend en compte les données du présent dans lequel on est ancré. Les facultés sensibles et intellectuelles sont exactement comme les facultés physiques. Si on ne fait pas de sport, on est moins musclé. Si on n'exerce pas son imagination, on en a moins. Si on n'exerce pas son intelligence, on en a moins, etc. C'est pour ça qu'il y a un caractère révolutionnaire à faire des films qui requièrent ces facultés chez nous. J.-M. S. - Simplement, il faut apprendre aux gens à voir et à entendre. S'ils ne sont plus capables de voir et d'entendre, ils ne sont pas capables de faire une révolution. La conscience commence par voir et entendre. J.-M. L. - Je suis à la fois d'accord et pas d'accord. J'aurais tendance à relativiser ce qui vient d'être dit. Ce qui me gêne un petit peu, c'est une forme de terrorisme. Une forme de terrorisme qui ferait que finalement, il y a des films qui sont faits pour voir et entendre, et des films qui ne le sont pas. Il y aurait une distinction assez nette entre le senti et le réfléchi, ce que je ne crois pas vraiment. Je suis assez d'accord avec la phrase de Walter Benjamin : il y a des émotions qui sont justes. C'est ce qui fait lien, par exemple, entre le cinéma classique et le cinéma contemporain. Ce n'est pas vraiment une question d'élaboration du discours qu'on pourrait porter sur les films ou de capacité à dire quelque chose des films. C'est ce qu'on ressent, finalement, ce que le film nous fait ressentir...

52 J.-M. S. - Je n'ai jamais prétendu qu'on était les seuls. Grâce à Dieu, on n'est pas les seuls! J.-M. L. - Oui, c'est ce que je trouve fort dans ton point de vue.

54 J.-M. S. - Le cinéma hollywoodien, pendant un certain temps, était une école de citoyens. Le cinéma japonais commercial, pendant un certain temps, était une école de citoyens. Comme on pourrait dire que la tragédie grecque était une école de citoyens. C'est classique, comme on dit!

J.-M. L. - Ça va mieux en le disant, quand même...

J.-M. S. - Ce n'est pas de ma faute si on me fait passer pour un terroriste isolé ! 
auteur? » Cela m'a fait penser à la notion d'énantiodromie chez Héraclite, qui parle de la loi fondamentale de la vie, selon laquelle chaque processus, à un certain moment, se renverse en son contraire. Arrivé à sa plus grande extension, il revient vers le centre. La discussion qu'on vient d'avoir sur l'Antiquité, les Modernes, les Classiques, etc. renvoie aussi à la question des grands cycles d'évolution, tant individuelle que collective. Après tout, il est difficile de dire comment les cycles collectifs et les cycles individuels se chevauchent. Lyotard a écrit un livre, en 1993, intitulé Moralité postmoderne, que certains connaissent certainement. Roger-Paul Droit, dans Le Monde, écrivait au sujet de ce livre : «Lyotard appelle donc moderne ce schéma de pensée qui assigne comme but ultime à l'histoire humaine de retrouver une perfection originaire devenue lointaine: Dieu dans la version chrétienne, la nature dans la perspective rousseauiste, la société sans classe dans la version marxiste. Le postmoderne, par 
contraste, serait donc comme le désenchantement de l'histoire. On continue d'agir, de négocier, de prévoir, mais l'ensemble du processus semble désormais dépourvu de toute finalité. Le développement suit son cours. Toutefois, il ne paraît plus destiné à parvenir à aucun terme. Les grands récits qui prétendaient donner sens à l'Histoire qu'il s'agisse des religions du livre ou de la confiance des Lumières dans le progrès, des analyse du marxisme - nous ont laissé en plan. Postmoderne est peut-être avant tout le nom d'une panne ». Donc, moderne serait une époque où les choses, où les mots et les choses tiennent encore un peu ensemble, dans un sens, dans une cohérence, qui était peut-être celle de l'Antiquité, etc., etc. Tout au moins, dans l'idée qu'on en a. Comment la retrouver, comment y revenir? C'est une question qu'on pourrait se poser très concrètement, en évitant de se perdre dans les mots. Comment, concrètement, y parvenir?

Spectateur - La piste égalitaire me semble bonne. Othon tire sa force de la possibilité de diviser. Je crois que vous avez dit que ce film divise le public - il divise le public en deux. Mais il peut aussi diviser chacun d'entre nous et, en même temps, nous libérer. Et c'est ça la modernité. C'est ça qu'a pensé la modernité. Le 2 plutôt que le 1 qui totalise. Quelque chose qui peut permettre de tenir, de faire tenir ensemble des choses hétérogènes.

Spectateur - Je voudrais juste faire une petite remarque. Concernant Corneille, moi, ce qui m'intéresse, c'est qu'on voit bien qu'en fait, pour Corneille, il ne s'agit pas de s'accorder sur le langage, il s'agit de s'accorder dans le langage. En fait, il me semble qu'il y a chez Corneille l'idée que la communauté de langage n'est pas fondatrice. C'est le but qu'on cherche à atteindre, mais elle n'existe pas a priori. Je pense que... j'ai l'impression que l'œuvre de Straub se retrouve dans cette approche de ce qu'est la communauté. À savoir une communauté qui n'existe pas a priori, mais qu'il faudrait peut-être essayer de créer, y compris à travers la dissonance. Ce qui m'intéresse, en fait, dans l'œuvre de Straub, ce n'est pas qu'elle est à contretemps, ou contre son époque. Je pense qu'au contraire, elle est complètement dans son époque, elle est dans son temps. Je crois même que cette cuvre fabrique son époque, en fait. Elle fabrique son temps. Comment elle le fait, comment elle fabrique son époque, cette ceuvre? Tout simplement à travers l'expression d'une voix dissonante. C'est-à-dire une voix qui prend confiance en elle-même, en fait. Qui cherche en tout cas à prendre confiance en elle-même. Cette fameuse confiance en soi dont certains philosophes américains parlent, comme Emerson. Je pense que c'est cette confiance en soi - self reliance -, ce processus de prise de confiance en soi que Straub nous relate. Et les difficultés que ça implique, bien sûr! Je pense aussi que cette prise de confiance en soi que vit le réalisateur, il veut la faire vivre au spectateur. C'est en ce sens qu'il y a pour moi quelque chose de très profondément démocratique dans le cinéma de Straub. Je voulais ajouter aussi qu'il me semble qu'il y a un désir, une puissance d'invention de la mythologie dans l'œuvre de Straub, face au constat de sa déflagration. Voilà.

65 D. F. - Je voulais juste repréciser ce que j'entends par contretemps, et qui n'est effectivement pas contradictoire avec ce que vous venez de dire. Contre le mouvement dominant des temps. Quand vous parlez de dissidence, évidemment, je ne peux qu'approuver. Ou de dissonance. Mais en ce sens-là, évidemment, Jean-Marie Straub est ancré dans son temps avec lequel il est dissonant. J'insistais aussi sur le fait que son cinéma est inséparable d'une analyse de ce dont le spectateur a besoin, ici et aujourd'hui. Parce qu'il n'est plus possible de faire aujourd'hui des films comme ceux du cinéma classique hollywoodien ou d'un certain cinéma japonais, qui étaient, comme le rappelait Straub tout à l'heure, une école de citoyen. Il faut en passer effectivement par des moyens plus radicaux, cinématographiquement s'entend, pour respecter ce 
postulat démocratique. J'aime beaucoup le discours de Pasolini qui vient d'être rapporté. Que c'est un signe de respect adressé au spectateur que de présupposer son intelligence, heureusement. Et donc, de la solliciter.

J.-M. S. - Quant à Pasolini, il faut quand même préciser quelque chose. Moi, je n'ai aucun respect pour Pasolini cinéaste. Mais aucun! J'ai pleuré en voyant Accattone à Munich avant d'aller en Italie. Et après ça, je ne veux pas dire... Je n'ai aucun respect. Autant je respecte Pasolini citoyen et Pasolini poète, autant je méprise Pasolini cinéaste. C'est tout. D'ailleurs, il ne me l'a pas envoyé dire, quand on se fréquentait, qu'on était un peu amis, il a écrit une dizaine de pages contre Othon. Et il a dit: Othon, c'est Straub qui enferme ses amis dans un camp de concentration. Point final. C'était l'idée. Il n'y en avait pas d'autre. Le camp de concentration, c'était la terrasse du Palatin. Rigolons sur Pasolini cinéaste, moi, je veux bien, mais enfin, il faut quand même dire les choses...

D. F. - Merci pour l'éclaircissement. Est-ce que je peux poser la question par rapport à Luchino Visconti ? La terre tremble, par exemple, qu'est-ce que vous en pensez ? J.-M. S. - Non, je ne veux pas donner des bons points.

D. F. - Il n'est pas question de bons points.

J.-M. S. - La version italienne doublée de La terre tremble est épouvantable. Ce n'est pas le pire film de Visconti. Mais malgré tout, je n'aime pas les entreprises populistes et je préfère Il Gattopardo. Il Gattopardo est dix fois plus émouvant que La terre tremble...

71 J.-M. L. - Est-ce que vous voulez encore intervenir?

72 J.-M. S. - Non, j'en ai assez.

\section{AUTEURS}

\section{DAVID FAROULT}

David Faroult est réalisateur et enseignant-chercheur en cinéma à l'Université Paris Est Marnela-Vallée. Il a soutenu un doctorat sur les groupes Cinéthique et Dziga Vertov, avant-gardes cinématographiques et politiques dans la France des années post-68.

\section{OLIVIER GOETZ}

Olivier Goetz est maître de conférences au département « Arts » de l'Université de Lorraine. Spécialiste en histoire et en esthétique des arts du spectacle, il développe une recherche transversale et pluridisciplinaire dans le domaine de la création artistique, des arts plastiques et des arts du spectacle.

\section{JEAN-MARC LEVERATTO}

Jean-Marc Leveratto est Professeur de sociologie de la culture à l'Université de Lorraine. Directeur du Laboratoire Lorrain des Sciences Sociales, il est le spécialiste de la sociologie de la consommation culturelle et notamment de l'industrie du spectacle (théâtre et cinéma). 